\title{
Software Design Framework for Healthcare Systems
}

\author{
Samir K Amin \\ Faculty of Computing and \\ Information Technology \\ Sohar University \\ Sultanate of Oman
}

\author{
Dinesh Kumar Saini \\ Faculty of Computing and \\ Information Technology \\ Sohar University \\ Sultanate of Oman \\ Research Fellow and Adjunct \\ Faculty, University of \\ Queensland Australia
}

\author{
Yazan S.K.Al-Gnabi \\ Institute of Micro engineering \\ and Nano electronics (IMEN) \\ National University of Malaysia
}

\begin{abstract}
Healthcare is costing lot of money and resources all over the world in all countries; it consumes almost 30-40 percent of their budget in the healthcare industry. The only solution is to make this health care industry automated and online. In this paper we are proposing a software design framework for health care systems which will be based on agent technologies. The proposed system is based on Artificial Intelligence techniques that can support the user for selection and making choices. The proposed software framework suggested that Multi-Agent Systems (MAS) is the most suitable technique for designing such systems.
\end{abstract}

MAS collaborate intelligently for solving this complex problem. Patients can be supported remotely using the proposed framework so that it can reduce the patient load on hospitals, the proposed software framework operate on real time framework. Computing can help in improving the communication process between follow-up doctors and nurses with patients by making appointments the process easier, according to patient preference with a reminder on necessary actions such as taking scheduled prescribed medicine, engaging in exercises, avoiding some kinds of food and harmful habits such as smoking before and after patient.

\section{General Terms}

Health Systems, Computing, Framework.

\section{Keywords}

Agent Technology, Multi Agent System, Communication Technologies, Health Informatics, Healthcare Process, Collaboration Technologies.

\section{INTRODUCTION}

This paper presents a Multi-Agent System (MAS) for supporting physicians in performing follow-up procedures remotely to patients. This system is designed to operate in a real-time scenario. Different agent-types of the MAS are introduced. Agents are designed to play a special role as representatives for human actors in the environment of clinical follow-up procedures [4]. Moreover the system comprises special administrator agents. A motivation for the use of agents will be given, as well as a specification of the problem, a description of design features, and the inter-agent communication structure that adopts a Collaborative Case Base Reasoning Using Implicit Culture Framework for analyzing patient's information and decision making.

Multi-Agent Systems (MAS) are capable of realizing a role oriented communication process in a clinical follow-up. The design and implementation of MAS is described, which should help to manage the course of a clinical follow-up.

Special attention is given to improving the communication process between follow-up doctors and nurses with patients by making appointments easier, according to the patient's preference with a reminder on necessary actions such as taking medicine, doing exercises, avoiding some kinds of food and harmful habits like such as smoking before and after patient visits.

The paper is organized as follows: Section 2 gives a general idea about the Framework of Healthcare process; Section 3 discusses the motivation of using agent technologies to support clinical follow-up procedure. Section 4 describes the problem specification of the task and shows the design of the MAS. Section 5 deals with the inter-agent communication and some implementation aspects. Finally, Section 6 closes with an outlook to future work and discusses the advantages and disadvantages of the approach.

\section{FRAMEWORK OF HEALTHCARE PROCESS}

Healthcare industry is heterogeneous in nature, it generate lot of information and data from patients, hospitals, pharmacies, Labs, ambulances, trauma care, doctors, relatives of the patients, insurance companies etc. It is very big and complex domain to bring all things on automated platform and online. So this sector need a proper software framework with all processes covered. These healthcare industries possess the most important characteristics which are as following:

It is prevalent that the knowledge required in solving a problem is spatially distributed at different locations, with different people and with different process.

The solution to a problem involves the coordination of efforts of different individuals with different skills and functions.

Medical care problems are quite complex, and finding standard software engineering solutions for them is not straightforward.

There is a great amount of medical knowledge available on the Internet. It is necessary to provide ways of accessing the most relevant information as easily, flexibly and timely as possible. This access to medical information is necessary both for the medical practitioners as well as for patients. In the former case, they have to be aware of all the latest and emerging medicines, techniques and treatments in their field of expertise; however, most practitioners lack the time to each for and filter through all these information and single out the appropriate and precise medicine/s that is/are needed. 


\section{Framework of Healthcare Process}

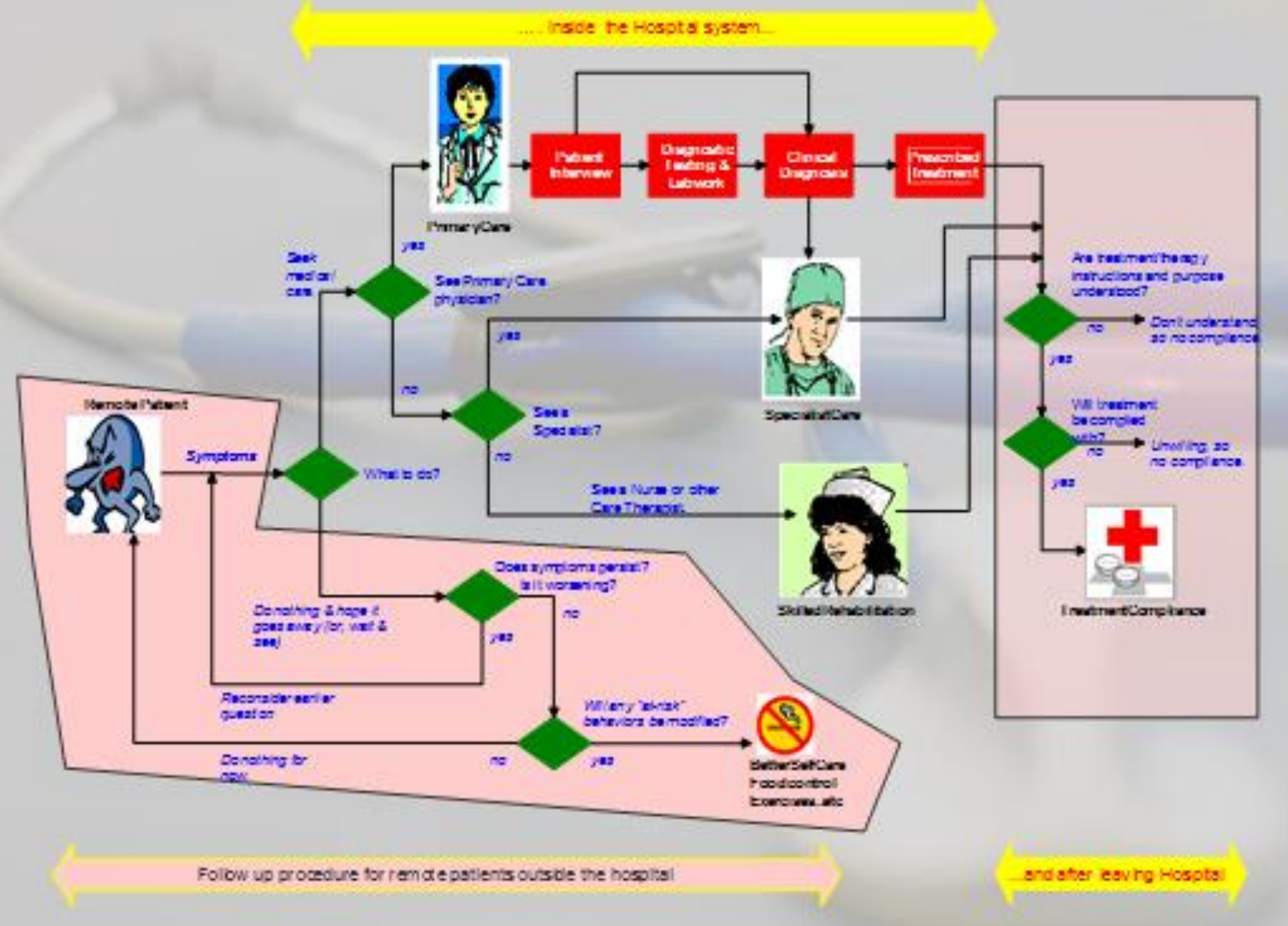

Figure1: The framework of healthcare process

In the latter, citizens demand a more active role in the management of their care, and they require ease of search for the relevant information (but they usually lack the skills to search for the most adequate information relevant to their specific personal needs). In both cases it is important to receive appropriate information from useful and reliable sources in a proactive way, without having to devote time and effort to look for, analyze, evaluate and filter it. Fig.1 shows the main three areas that any healthcare process must concentrate, work on, and address upon, these are namely the remote patient (before coming to hospital), inside the hospital systems and after leaving the hospital (follow-up). Our work is to support physicians in performing remotely follow-up procedures to patients by using a Multi-Agent System (MAS) technology that runs on the utilization of mobile devices. In order to establish the necessary communications for this purpose we will be using applications that can be run using ordinary mobile phones. 3. Multi-agent systems in medicine

\section{AGENT TECHNOLOGIES}

Most of the emerging latest software frameworks uses an agent technologies which is based on Artificial Intelligence techniques to choose the best set of actions to perform in order to reach a goal specified by the users, which are patients and doctors. Agent technology is flexible, proactive, dynamic, autonomous and intelligent in nature and proved to be very way to the changes produced in its environment. A multiagent system may be defined as a collection of autonomous agents that communicate between each other to coordinate their activities in solving collectively any problem that could not be tackled by any other agent individually.

In recent years multi-agent systems have been considered as the latest software engineering paradigm. This kind of systems may be used in domains with the following features:

That knowledge is distributed in different locations.

That possesses several entities, while keeping their autonomous behavior, whereby the MAS system will have to join their problem-solving abilities to be able to solve a complex problem.

he problems in the domain may be decomposed in different sub-problems, even if they have some kind of interdependencies.

There is a growing interest in the application of agent-based techniques to problems in the medical domain. Multi-agent systems are indeed an interesting tool to solve such problems, since the usual properties of intelligent agents match quite precisely with needs in this field (basically with the requirement of having autonomous intelligent proactive collaborative entities in a distributed environment). 
Whereas the introduction of techniques of Artificial Intelligence in medicine, for example; rule-based expert systems to support decision processes, has a long term history, whereas the development of MAS in medicine and health care is a comparable young field.

To enable the modelling, designing and building of complex software systems in medical domains requirements such as distribution, flexibility, cooperation, autonomy and scalability, agent technologies seem to be a perfect to match the requirements and oversee these constraints. In view of this, there has been a growing interest in the application of agent-based systems in the health care industry in the last five years. Some specialized workshops in this topic have begun to appear [13], [2], [1]

Scheduling tasks in hospitals have utilized prototypical MAS in current day scenario. Distributed patient scheduling with respect to appointments in different functional units within a hospital, [11], [3].

The coordination of patient scheduling in hospitals for the efficient management of medical processes is considered in [5] and the administration of shifts in a hospital and its subdepartments is part of the INKA project [10].

In the MAS "ChariTime" of [11] there are two types of agents; the Knowledge agent and the User agent. But for every agent type there has to be installed a number of instances depending on the number of organizational units of a hospital on the one hand and the number of users into the system on the other hand. From the system design point of view the number of alive/active agents at one time is unlimited.

The ADAPT [5], [6] project is cited here as an example of the usage of multi-agent techniques in the field of clinical studies. The system was developed to find out, characterize, model, plan, simulate and increase the efficiency of clinical processes in a hospital. Within this project clinical trials scenario was discussed to illustrate the support of clinical process management. A simulation of trial scheduling with respect to the coordination of patients, medical staff and equipment was executed. The aim was to find out which resources would be needed and which amount of costs will appear when a (special) clinical study would be carried out. The result should help the responsible clinicians to decide whether they should perform the trial or not.

Whereas ADAPT allows the act of reflecting, modelling and simulating clinical processes as a whole. The approach discussed in this paper focuses on the scheduling of patient behaviour in clinical trials to optimize these processes. In contrary to ADAPT, where only simulation is used as basis, the system presented in this paper works in a real-time environment.

Workflow Management Systems (WFMS) are currently introduced into clinical routine to manage established clinical pathways on a daily routine basis within a clinical setting with limited communication to outside entities [16]. The proposed MAS focus on communication with patients that are living outside the clinic. However, a detailed comparison between the advantages and disadvantages of WMFS and multi-agent techniques is not topic of this paper
Problem Specification, Design and Description of the underlying Multi-Agent System

In a remote clinical follow up procedure both information technology and Multi-Agent Systems are used to improve the care given to patients. In such procedures, critical timewindows exist in terms of the performance of an action such as taking medicine, coming to the hospital for examinations, hospitalization and doing some tests by the patient themselves and sending the results to the hospital. Not using the given timeframe can lead to an exclusion of patients from the ongoing clinical procedure and may be detrimental and harmful to the patient's health. Most reasons for 'nonperformance' can be found in the behaviour of the involved patients. A simple reason for these occurrences could be that a patient has forgotten or has miss-scheduled an appointment. Furthermore, a patient might miss taking medicine/prescriptions in a given time-window or misinterprets the guidelines for executing some tests. It is also possible that a patient was not or not correctly informed about the deferent actions to be taken by him from the clinical side.

The mentioned problems are often caused by an incomplete overview about the current state of each individual patient during the clinical follow-up and normally these problems in practice are determined too late. Agent technology, especially, multi-agent systems appear as a proper technique to prevent such events.

In this paper, our aim is to supervise these patient-relevant interactions during the clinical follow-up process. In order to realize this, the following MAS-structure was developed.

The MAS consists of six types of specialized agents (shown in Fig. 1). These agents represent human actors in the real life/real world environment of clinical follow-up, and are also additional relevant MAS-components. The usual number of patients in a clinical follow-up varies from one hundred up to thousands. For this reason the MAS must be flexible with respect to the number of involved agents. It is adaptable and scalable and is able to be utilised in various communication methods for human-machine-interaction.

\section{COMPONENTS}

\subsection{Supervisor}

The supervisor is a special agent, the 'Brain of the MAS'. There is only one supervisor at a time. It starts all other agents (startAgent()) and is able to terminate agents at any time (terminateAgent()). Only the supervisor has the right to request a report of all other agents about their status at any given time (reportStatus()). It has the knowledge about all active agents and synchronizes the whole MAS if needed. It does not interfere into medical matters. The only interaction to the human environment consists of contacting the system administrator in emergency cases.

\subsection{Patientagent}

Each patient in a clinical follow-up is represented in the MAS by an own patientagent. It watches all time related events during the participation of the patient in the follow up process course and points out possible future or recorded emergency cases and if possible suggests a solution to matters concerning the patient. 


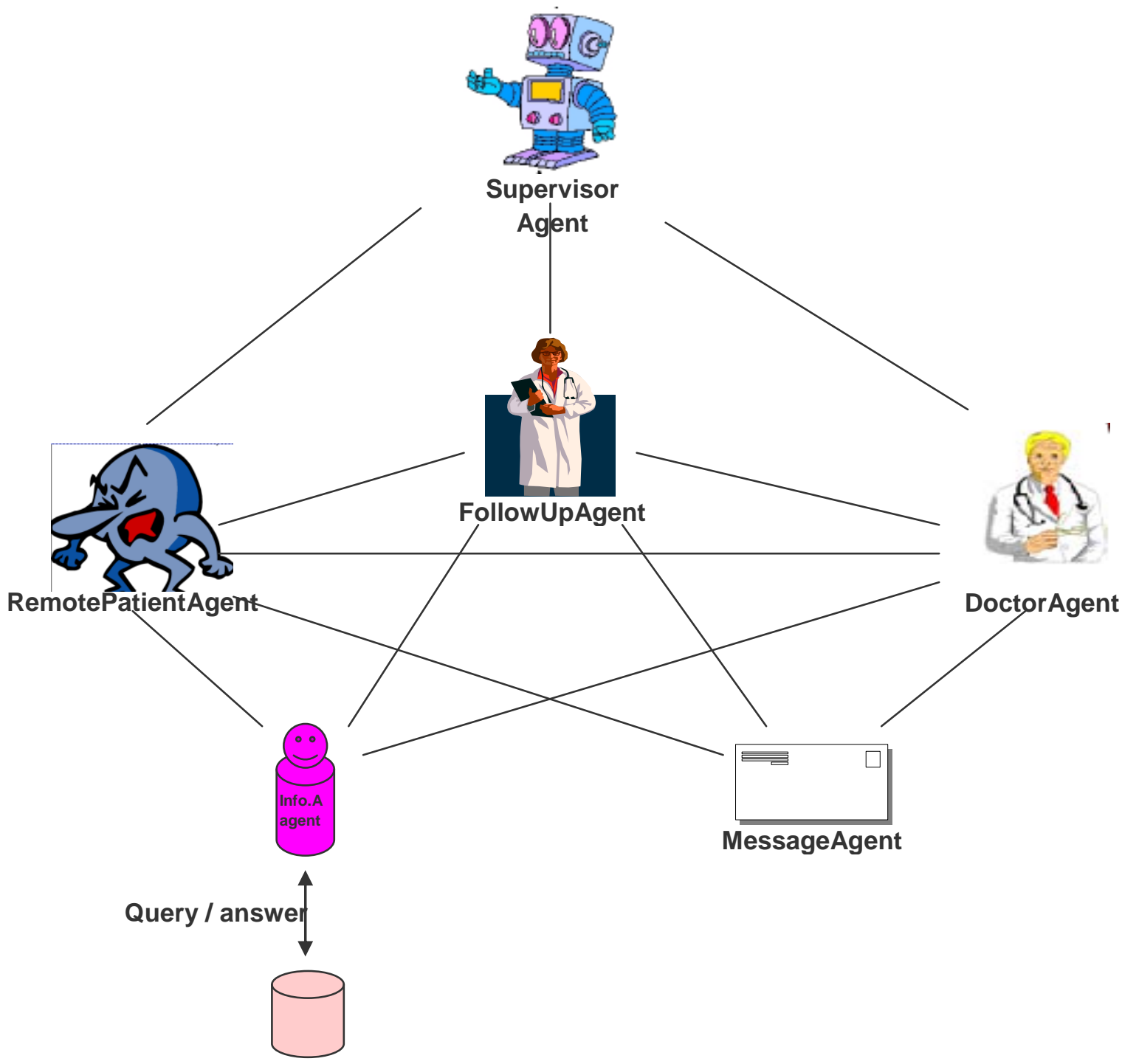

Figure2: Underlying Multi-Agent System

The agent contacts its patient via email and/or GSM messages (informPatient()) and reminds or requests the person of situations such as:

$>$ Nearby future appointments.

$>$ Reminder for taking or application of medications/prescriptions.

$>$ Preferences for future appointments.

$>$ Reminder/Request for medical reading or test results...etc

It persists in its attempts to contact its patient until a confirmation of the patient is received. If the patient does not reply in a given time interval the patientagent contacts the follow-up person (informFollowUpPerson()) and requests for help. The patientagent also constantly verifies if the time-schedule of the patient is in conformity with the given time schedule of the clinical follow-up. If a problem occurs it alerts the followupagent and doctoragent (informDoctorAgent()) and initiates the emergency procedures of them. It is achievable as long as the patient has active agent in the follow-up course.

\subsection{FollowUpAgent}

Each followupagent is linked to one specific follow-up process (there may be many follow-up processes depending on types of patient's case) which is performed in the clinic. The followupagent receives messages from the patientagents, analyses them by using case-based reasoning and passes information to a doctoragent (informDoctorAgent()) or contacts a follow-up person (informFollowUpPerson()) by email when human help is needed. For example, a phone-call to a patient is necessary to ensure the patient's participation in an examination in the next days. The followupagent is alive as long as the follow-up process is running/going on. 


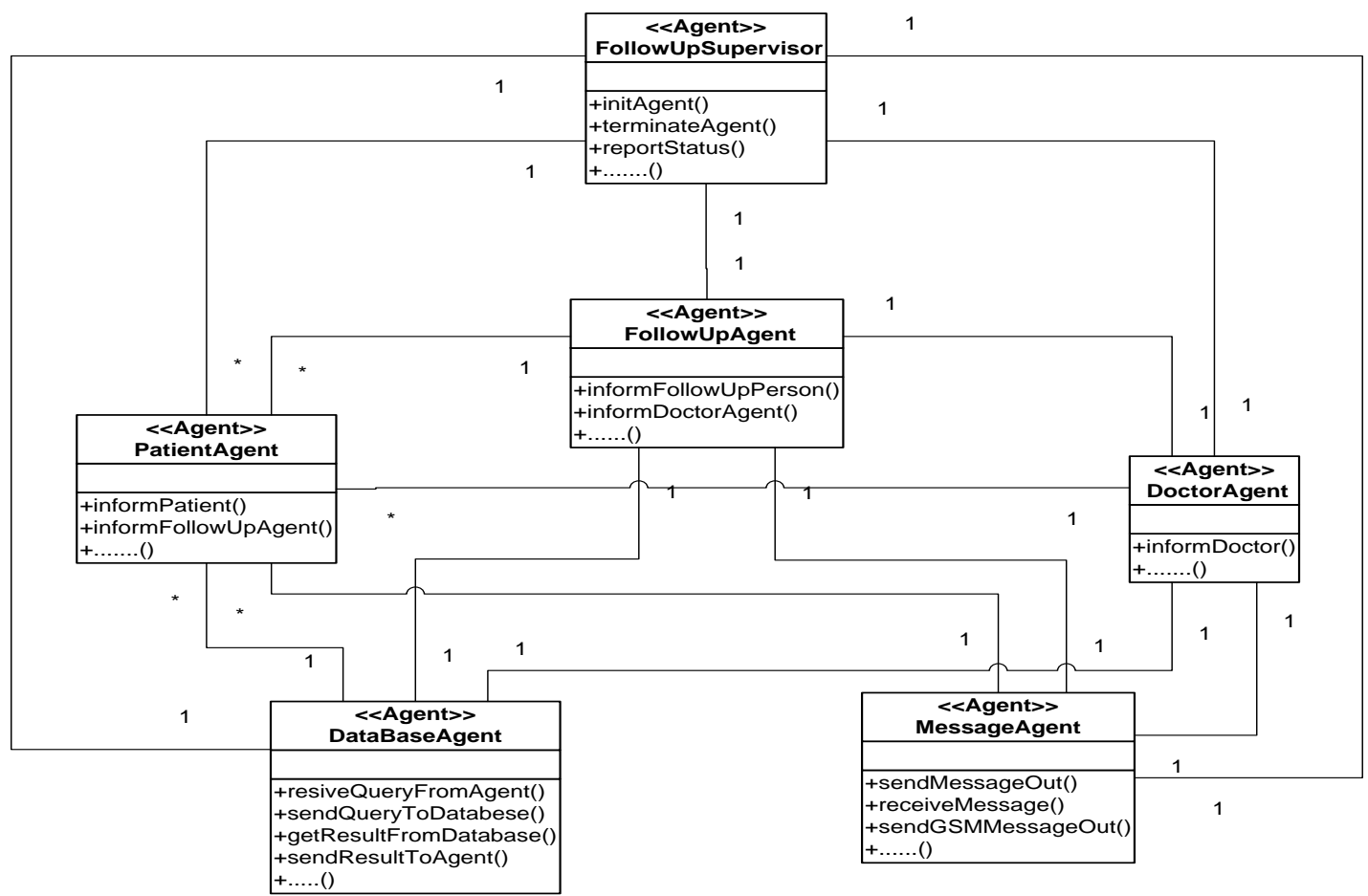

Figure3: Core Agents for follow up process

\subsection{Doctoragent}

Each doctoragent is dedicated to one specific physician of the clinic. It redirects all relevant data to the doctor per email (informDoctor()), where the doctor has the option to set filterrules for time-intervals when information shall be given (once an hour, once a day ... ) and what kind of information is of interest (all, only high prioritized ...). The doctoragent could receive the information from several followupagents (not displayed in Fig. 2; only one clinical follow up is shown there) pertaining to their analysis of the patient's case and of course from a lot of patientagents too. This agent-type is alive for every physician as long as the doctor is a member of the clinical follow-up.

\subsection{Databaseconnector}

The databaseconnector is the only agent in the MAS that is allowed to communicate with the underlying MySQLDatabase. It is able to receive queries (written in SQL-code) from all other agents (receiveQueryFromAgent()) and passes these to the database (sendQueryToDatabase()). The results (getResultFromDatabase()) will then be send back to the requesting agent (sendResultToAgent()). This structure was chosen to prevent transaction-errors or others that could take place by simultaneous agents accessing on the database.

\subsection{Messageagent}

Similar to the databaseconnector that is the only agent which communicates to the database, the messageagent is the only agent in the MAS that is really able to communicate with humans. All messages to the patients, to the follow-up person and to the doctor are sent by this agent via emails (sendMessageOut()) and/or GSM messages (sendGSMMessageOut()) and all received answers (receiveMessage()) are passed to the right agent. This agent has a small dictionary that enables it to translate its messages and recommendations into Arabic language for persons who do not understand English. The advantage of this structure is its adaptability. If new ways of communications are chosen (e.g. sending messages through Voice over IP) or as in our case, communicating with an application running on mobile phones, only this agent must be adapted to this new form of communication, but all other agents can stay untouched. Email and GSM messages were chosen as the possession of mobile phones or email account are the most common denominator that many patients possess. Fig. 3, illustrates the way this agent communicates with applications running by laptops or mobile phones. Fig.4. indicates some of the screens of a mobile phone application with regards to Messageagent mobile phone user end interface, while Fig. 5 


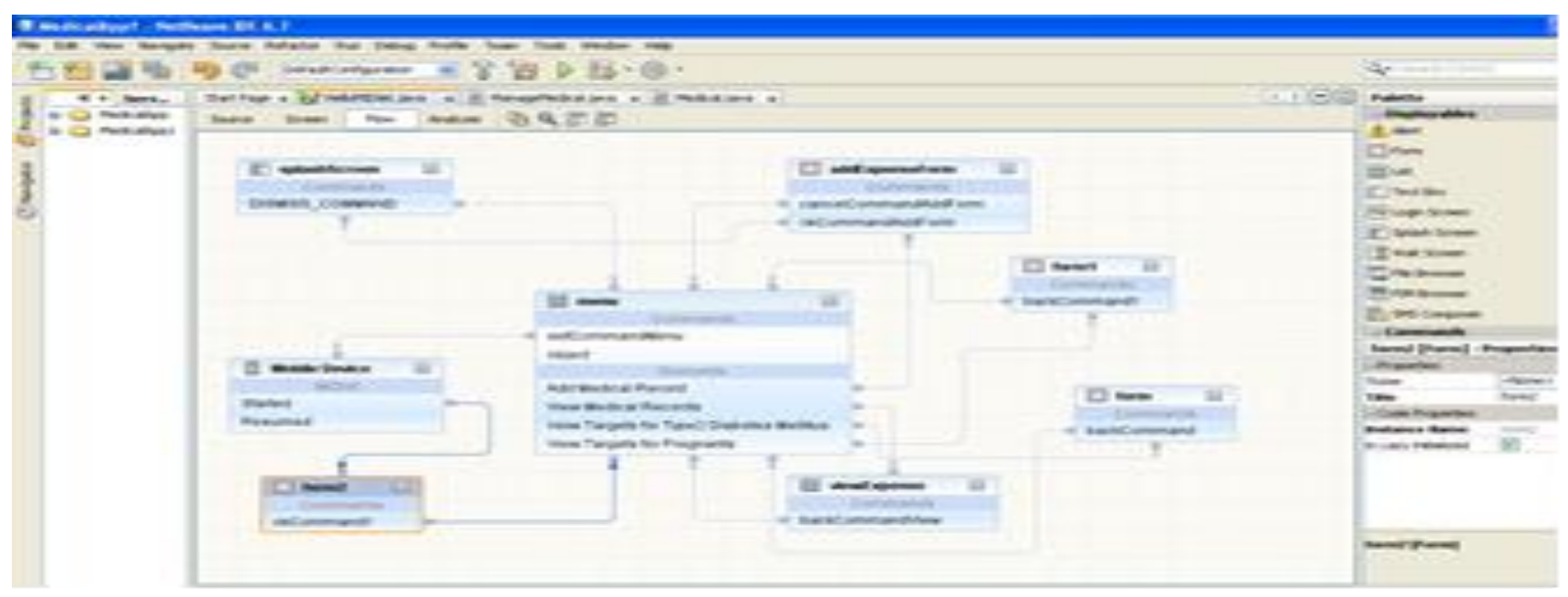

Figure4: Screenshots of the systems

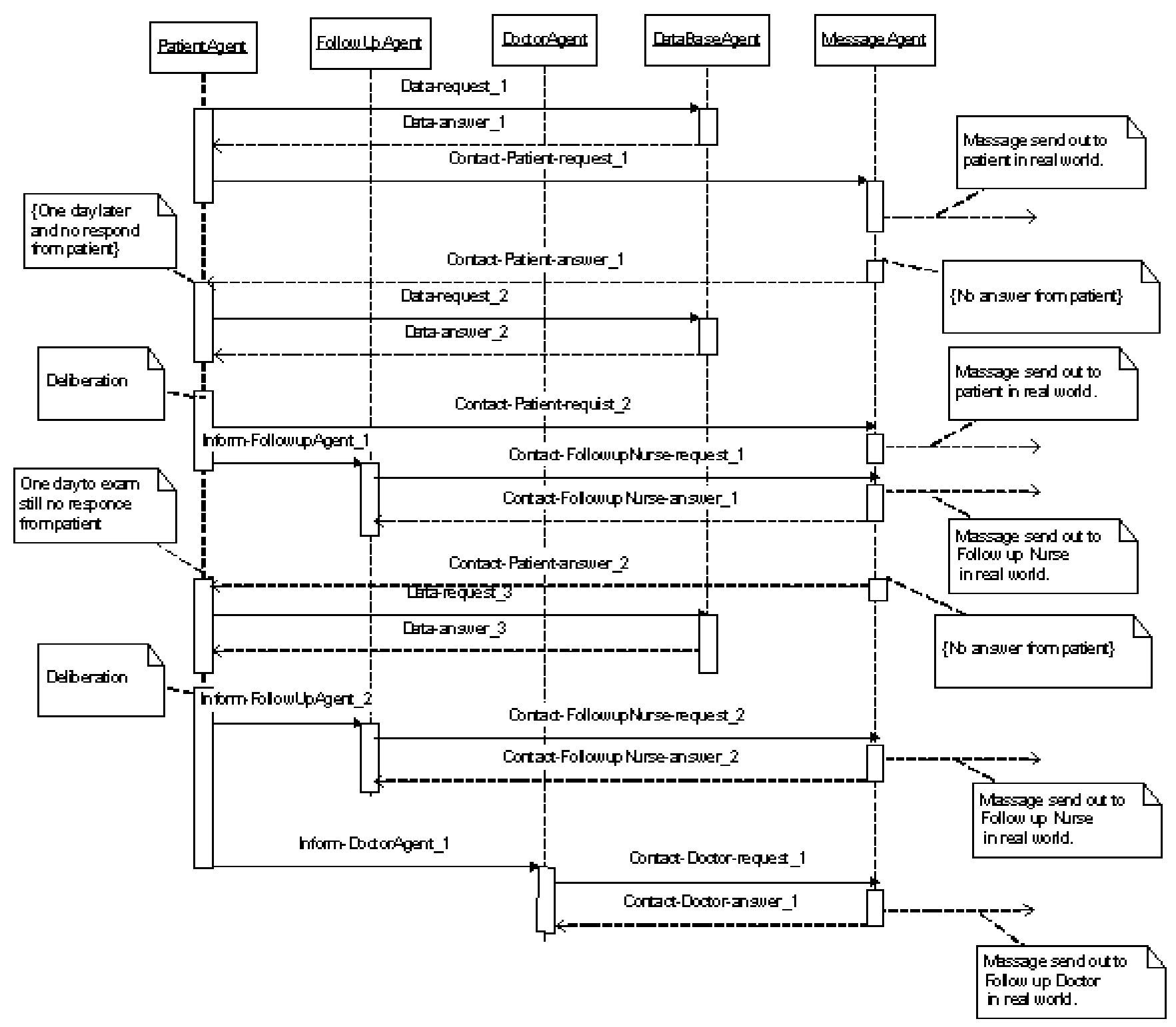

Figure5: Inter-agent communication and implementation aspect 


\section{COMMUNICATION}

The following section describes a special inter-agent communication case which depicts a situation in the event that a patient does not respond to appointment requests. As shown in Fig. 5 the patientagent is mainly the initiating agent of the agent communication in the MAS.

After it receives the latest information regarding the status of the patient (Data_request_1, Data_answer_1) from the database agent, it deliberates these information. If the followup time schedule demands a new examination in the next few days, the message agent will be contacted (Contact_Patient_request_1) to send out an email to the patient reminding of the appointment including a request for an answer. One day later the message agent informs the patient agent (Contact_Patient_answer_1) in the event that there is no answer received yet from the patient. After a new information update (Data_request_2, Data_answer_2) the patient agent decides to repeat the patient-email (Contact_Patient_request_2) and to inform the follow-up nurse of a possible problem with the next patient visit (Inform_Followupagent_1). The follow up agent receives this, interprets it as an important information and sends it to the follow-up nurse over the message agent via email, so that the follow-up nurse can decide if there is the need perform an action (e.g. a phone call) by (Contact_FollowUpNurse_request_1, Contact_FollowUpNurse_answer_1).

If on the examination day there is still no answer from the patient (Contact_Patient_answer_2), then as a last resort by the patient agent, it informs both the study nurse and the doctor, that the patient will most likely miss the appointment for this day (InformStudyAgent_2, InformDoctorAgent_1).

In this case the study agent and the doctor agent decide that this information is important enough to inform the study nurse and the doctor as well (Contact_StudyNurse_request_2, Contact_Doctor_request_1). Now the clinical staff is fully aware of the problem and can initiate emergency procedures (e.g. further phone calls, re-scheduling of the examination ...) if needed.

As agent architecture the Belief-Desire-Intention-paradigm (BDI) [12] was chosen. With help of BDI it is possible to create intelligent agents which are capable of performing long time term plans and which are able to adapt them to changing environments.

The basis/foundation of the implementation of the above mentioned multi-agent system is the Java-Agent Development-Environment (JADE) [JADE 2009]. JADE is now the quasi-standard platform for java-based agentsystems. It provides a well operating structure for inter-agent communication and by this enables the developer to create a working MAS within a short period of time. JADE completely supports the FIPA-ACL standards [4].

JADEX [JADEX 2009], the JADe Extension, introduces new features into JADE, which support the BDIparadigm. Believes, Desires and plans (Intentions) - BDI, can easily be created and combined. A simple MySQL-database is used to support the base-functionalities of the agents. In regular intervals (e.g. once a day) this database receives updates from other medical systems (provided by Siemens Medical Solutions)[17]. This latest information mode a pertaining to new patient appointments, new medical treatments and others are introduced into the MAS. Fig. 6 shows the opening screen for JADE Remote Agent Management GUI.

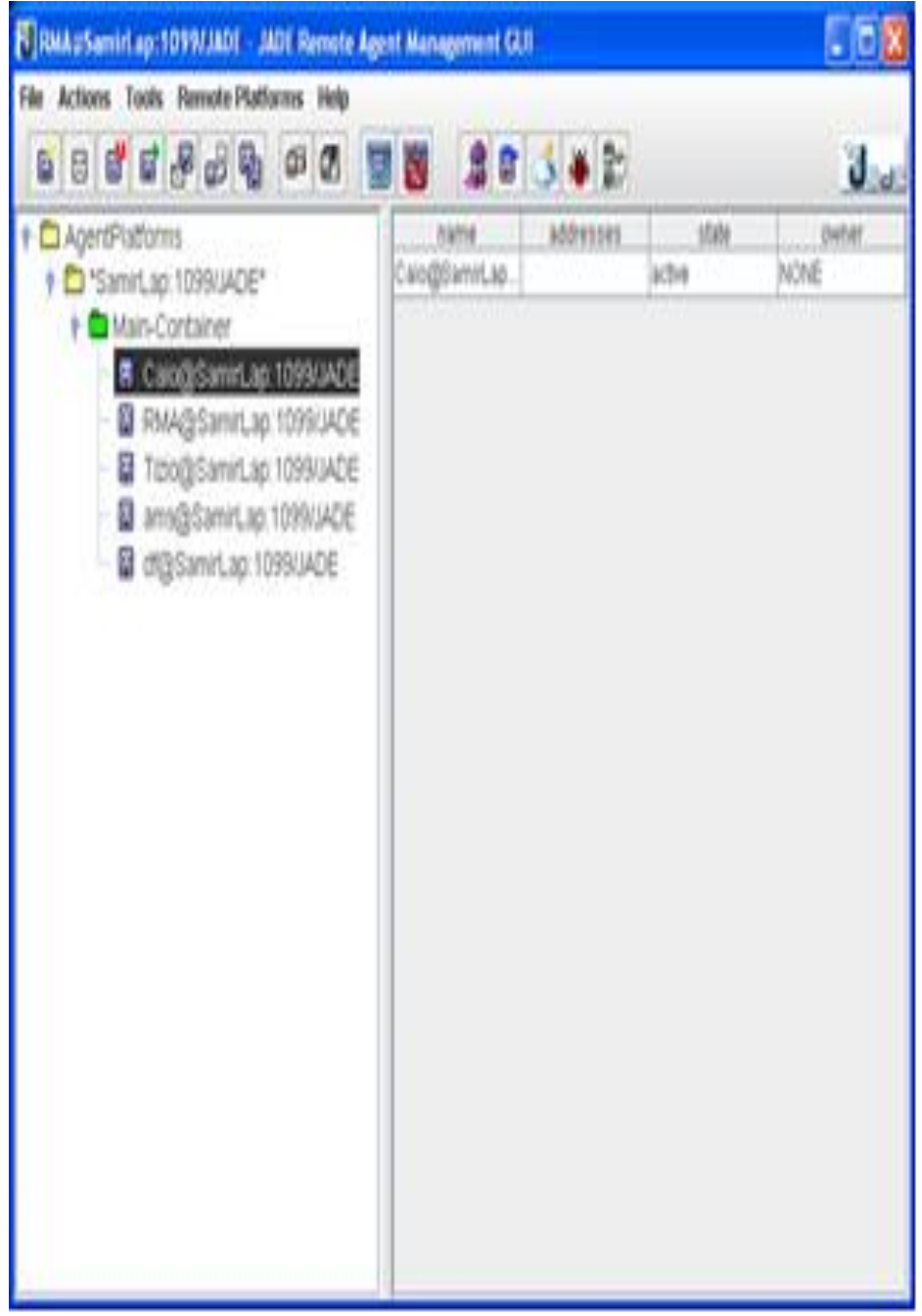

Fig6. JADE Remote Agent Management GUI

\section{CONCLUSION}

Agent technologies are rapidly gaining pace in health informatics and proved to be very useful in the designing health informatics systems. Multi agent systems are becoming proactive and intelligent in terms of health data and clinical follow up. MAS which are capable of realising a role oriented communication process in a clinical follow up. The design and implementation of such MAS is described, which should help to manage the course of a clinical follow up. Special attention is given to improving the communication process between follow-up doctors and nurses with patients by making appointments easier, according to patient preference with a reminder on necessary actions such as taking scheduled prescribed medicine, engaging in exercises, avoiding some kinds of food and harmful habits such as smoking before and after patient visits. Health informatics is ongoing developing field of research and MAS has solid role to play. With the MAS systems in place we can reduce the load on hospitals and can facilitate the doctors in follow up of the patients.

\section{REFERENCES}

[1] ARMANO ET.AL., 2005. Editors: Proceedings Workshop MAS*BIOMED, International Conference AAMAS 2005, Utrecht, The Netherlands, 2005.

[2] Cortes U., Fox J., Moreno A., 2002. Editors: Proceedings Workshop on Agents Applied in Health Care, in the 15th European Conference on Artificial Intelligence, ECAI- 
02. Lyon, France: IOS Press; July 2002.

[3] Decker K., Li J 1998.Coordinated hospital patient scheduling, Proceedings of the Fourth International Conference on Multi-Agent Systems, ICMAS-98. Paris, 1998.

[4] WM Omar, DK Saini, M Hasan "Credibility of Digital Content in a Healthcare Collaborative Community", Software Tools and Algorithms for Biological Systems, pp.717-724 USA 2012.

[5] Heine C., Herrler R., Petsch M., Anhalt C.: ADAPT Adaptive Multi Agent Process Planing \& Coordination of Clinical Trials, in: Proceedings of AmCIS2003, Tampa, Florida, USA, 2003

[6] Heine C., Herrler R., Kirn S.: ADAPT@ Agent.Hospital: Agent-Based Organization \& Management of Clinical Processes, International Journal of Intelligent Information Technologies, Vol. 1, No. 1, 2005

[7] N Gupta, D Saini, H Saini "Class Level Test Case Generation in Object Oriented Software Testing", Web Engineering Advancements and Trends: Building New Dimensions of Information Technology,

[8] http://vsis-www.informatik.unihamburg.de/projects/jadex

[9] Lindemann G., Münch I., Schulz-Schaefer I.: An Approach to Model Hybrid Organizations in the Context of Appointment Management, in: Proceedings of the Workshop MASHO, ECAI2000, Berlin, 2000

[10] Meister M., Urbig D., Schreter K., Gerstl R.: Agents Enacting Social Roles. Balancing Formal Structure and Practical Rationality in MAS Design. In: Florian,
Michael/Klaus Fischer (editors.): Socionics: Its Contribution to the Scalability of Complex Systems. Berlin, Heidelberg. Springer (Lecture Notes in Artificial Intelligence (LNAI 3413), 2005

[11] Münch I., Lindemann-v. Trzebiatowski G.: ChariTime Concepts of Analysis and Design of an Agent-Oriented System for Appointment Management. fundamental Informaticae, Vol. 43, IOS Press, The Netherlands, 2000

[12] Rao A., Georgeff M.: Modeling rational agents within a BDI- architecture. In: Proc. of the 2nd Int. Conf. on Principles of Knowledge Representation and Reasoning (KR-91), San Mateo, CA, Morgan Kaufman Publishers, 1991.

[13] Shankararaman V., editor: Proceedings of the Workshop on Agents in Health Care, in the 4th International Conference on Autonomous Agents, Agents-00. Barcelona, Spain: ACM Press; June 2000.

[14] http://www.medical.siemens.com.accessed on Oct.2014.

[15] Helmut Myritz ,Gabriela Lindemann ， Gudrun Zahlmann: Paper "Patient Scheduling in Clinical Studies with Multi-Agent Techniques , 2006.

[16] J. Tweedale, N. Ichalkaranje, C. Sioutis, B. Jarvis, A. Consoli, G. Phillips-Wren " Innovations in multi-agent systems" Journal of Network and Computer Applications, Volume 30, Issue 3, August 2007, Pages 1089-1115

[17] Dante I. Tapia, Juan A. Fraile, Sara Rodríguez, Ricardo S. Alonso, Juan M. Corchado "Integrating hardware agents into an enhanced multi-agent architecture for Ambient Intelligence Systems" Information Sciences, Volume 222, 10 February 2013, Pages 47-65. 